\title{
Análisis e implicaciones de los resultados de las prácticas pedagógicas de un docente líder en una prueba piloto de innovación en el proyecto Conectándonos
}

\author{
Analysis and Implications of the Results of a Teacher Leader's Pedagogical Practices in a \\ Pilot Test on Innovation in Conectándonos Project
}

\section{Análise e implicações dos resultados das práticas pedagógicas de um professor líder em um teste piloto de inovação no projeto Conectando-nos}

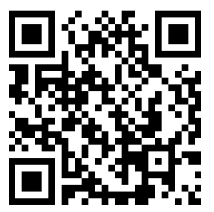

Manuel Baltodano-Enríquez ${ }^{1}$

Universidad Estatal a Distancia

San José, Costa Rica

mbaltodano@uned.ac.cr

iD http://orcid.org/0000-0003-2599-2192

Recibido • Received • Recebido: 25 / 06 / 2016

Corregido • Revised • Revisado: 18 / 01 / 2018

Aceptado • Accepted • Aprovado: 13/ 02 / 2018

\begin{abstract}
Resumen: Este artículo presenta los resultados de las prácticas pedagógicas de un docente líder en una prueba piloto de innovación desarrollada en una escuela primaria de Costa Rica. La prueba piloto se efectuó por la necesidad de cambiar el equipo tecnológico en los centros escolares pertenecientes al proyecto Conectándonos MEP-FQT del Departamento de Investigación, Desarrollo e Implementación del Ministerio de Educación Pública y de la Fundación Quirós Tanzi. Esta prueba piloto consistió en diagnosticar las prácticas pedagógicas de un docente líder mediante la exploración y el uso de recursos didácticos digitales contenidos en las computadoras portátiles tipo Clamshell y un proyector interactivo. Los equipos fueron utilizados para favorecer la mediación pedagógica del docente mediante el acceso, la selección y el uso de recursos didácticos digitales. Para la recolección de los datos, se utilizaron guías de observación no participante, un cuestionario con preguntas abiertas y la revisión documental del planeamiento didáctico del docente. La sistematización de las guías de observación permitió evidenciar de manera natural y concreta los aspectos contemplados
\end{abstract}

${ }^{1}$ Doctor en Ciencias de la Educación con Mención en Administración Educativa. Máster en Tecnología Educativa con Acentuación en Capacitación Corporativa y Formación Profesional, Escuela de Educación, Humanidades y Ciencias Sociales del Instituto Tecnológico y de Estudios Superiores de Monterrey. Profesor de investigación en la Escuela de Ciencias de la Educación de la Universidad Estatal a Distancia. 
doi: http://dx.doi.org/10.15359/ree.22-2.4

URL: http://www.una.ac.cr/educare

CORREO: educare@una.cr

en los objetivos del estudio. El análisis de la información se realizó mediante un enfoque cualitativo de tipo fenomenológico, donde se definieron, categorías y subcategorías que posteriormente, se vincularon entre sí, para establecer coincidencias, similitudes, diferencias, presencia o ausencia de términos o frases. Asimismo, se realizó una interpretación de los datos, donde se relacionó la información obtenida mediante el análisis y los referentes teóricos que permitió, posteriormente, reflexionar sobre las categorías estudiadas. Por último, como conclusión principal de la prueba piloto, se deriva la promoción del trabajo colaborativo entre estudiantes y el logro de los objetivos de aprendizaje propuestos en el planeamiento didáctico elaborado por el docente.

Palabras claves: Tecnologías digitales; mediación pedagógica; habilidades tecnológicas; comunicación digital; gestión del conocimiento.

Abstract: This article presents the results of a teacher leader's pedagogical practices in a pilot test on innovation implemented in a Costa Rican primary school. The pilot test was conducted due to the need of renewing the technological equipment in schools belonging to Conectándonos MEP-FQT project run by the Department of Research, Development, and Implementation of the Ministry of Public Education and Quiros-Tanzi Foundation. This pilot test aimed to diagnose the pedagogical practices of a teacher leader through the exploration and use of didactic digital resources of Clamshell-type laptops and an interactive projector. This equipment was used to favor the teacher's pedagogical mediation through the access, selection, and use of didactic digital resources. To collect the information, we used non-participant observation guides, a questionnaire with open questions, and a bibliographic review of the teacher's didactic planning. The systematization of the observation guides permitted to put in evidence, naturally and concretely, the aspects covered by the objectives of the study. The information was analyzed with a qualitative approach of phenomenological type. This approach defined categories and subcategories that, later, were linked to establish coincidences, similarities, differences, and the presence or absence of terms and phrases. Likewise, the data were interpreted to relate the information collected through the analysis and the theoretical patterns. This interpretation permitted, afterwards, to reflect on the studied categories. Finally, the main conclusion drawn from the pilot test was that the collaborative work between students was promoted and the learning objectives in the didactic planning drawn up by the teacher were achieved.

Keywords: Digital technologies; pedagogical mediation; technological skills; digital communication; knowledge management.

Resumo: Este artigo apresenta os resultados de práticas pedagógicas de um professor líder em um teste piloto de inovação desenvolvida numa escola primária em Costa Rica. O teste piloto foi realizado pela necessidade de mudar o equipamento tecnológico nas escolas pertencentes ao projeto Conectando-nos MEP-FQT do Departamento de Pesquisa, Desenvolvimento e Implementação do Ministério de Educação e da Fundação Quirós Tanzi. O teste piloto consistiu em diagnosticar as práticas pedagógicas de um professor líder através da exploração e do uso dos recursos didáticos digitais que trazem os computadores laptops e um projetor interativo. Os equipamentos foram usados para promover a mediação pedagógica do professor através do acesso, seleção e utilização dos recursos didáticos digitais. Para coletar os dados, foram usados guias de observação não participante, um questionário com perguntas abertas e a revisão de documentos de planejamento didático do professor. A sistematização das guias de observação permitiu comprovar de forma natural e concreta 


\begin{abstract}
os aspectos abrangidos pelos objetivos do estudo. A análise dos dados foi realizada utilizando uma abordagem qualitativa fenomenológica, onde foram definidas categorias e subcategorias que posteriormente foram relacionadas entre si, para estabelecer coincidências, semelhanças, diferenças, presença ou ausência de termos ou frases. Além disso, realizou-se uma interpretação dos dados, onde as informações obtidas através da análise e do referencial teórico se relacionaram, permitindo assim uma reflexão posterior sobre as categorias relacionadas. Finalmente, a principal conclusão do teste piloto consiste na divulgação do trabalho colaborativo entre os estudantes e a realização dos objetivos de aprendizagem propostos no planejamento educacional do professor.
\end{abstract}

Palavras-chave: Tecnologias digitais; mediação pedagógica; competências tecnológicas; comunicação digital; gestão do conhecimento.

\title{
Introducción
}

En los sistemas educativos mundiales existe consenso en reconocer la necesidad de incorporar las tecnologías digitales (TD) como medios para favorecer la innovación educativa e incidir positivamente en los procesos administrativos, actividades de proyección e integración comunal, el aprendizaje y la socialización del estudiantado, la atención a las necesidades educativas especiales, la formación, y las prácticas de enseñanza del profesorado, entre otros (Fallas y Trejos, 2013; Ministerio de Educación Pública, 2010; Unesco, 2004).

En este estudio se hace mención a las TD considerando un espectro más amplio de acción que el que subyace en el concepto de tecnologías de la información y la comunicación, conocidas popularmente con el acrónimo de TIC.

Desde la perspectiva pedagógica, la información es vista como contenidos informativos que se trasfieren o comunican para el logro de los aprendizajes, sin embargo, la simple trasferencia de información y la comunicación no genera ningún conocimiento (Fonseca 2000; Resnick, 2000). Un ejemplo clásico de tecnologías de la información y la comunicación lo constituye la radio y la televisión que generaron grandes expectativas cuando fueron introducidas como recursos didácticos para el aprendizaje en los contextos escolares; sin embargo, a lo largo de los años no mostraron mayor incidencia en la trasformación de las estrategias para la enseñanza y el aprendizaje.

Por ello, en este caso se concibe la tecnología como un recurso didáctico que no solamente coadyuva a la simple trasferencia de información y la comunicación, sino que actúa como un medio para el diseño y la creación de conocimiento por medio del internet y los recursos digitales de la Web, y esto se logra cuando la tecnología permite trascender de la simple emisión y recepción de información, a la interactividad y trasferencia bidireccional entre dispositivo y estudiante. En relación con lo anterior, es lo digital de la tecnología lo que "...produce un cambio cuántico, abre las puertas a la interactividad, los hipermedios, la realidad virtual, la telepresencia, la robótica, la simulación, el correo electrónico y la vida en línea" (Fonseca, 2002, p. 4). Son las TD quienes 
doi: http://dx.doi.org/10.15359/ree.22-2.4

URL: http://www.una.ac.cr/educare

CORREO: educare@una.cr

permiten la activación de "... elementos inteligentes en el punto de origen y en el punto de destino. Lo digital hace posible la superación del paradigma de la 'emisión' o 'broadcasting' que caracterizó las tecnologías anteriores, particularmente la radio y la televisión" (Fonseca, 2002, p. 4).

Por lo antes descrito, en este artículo se hará referencia a las TD y no a tecnologías de la información y la comunicación.

En la actualidad, las tendencias mundiales de integración de TD apuntan a la flexibilización de los procesos y los espacios educativos. Estas tendencias han sido potenciadas, gracias al desarrollo de dispositivos móviles inteligentes, que permiten una fácil portabilidad, acceso a la información mediante conexiones inalámbricas e interacción de los actores en entornos virtuales de aprendizaje. Asimismo, se han conformado grupos de público usuario en espacios interactivos que permiten el uso integrado de las TD en el currículo, las prácticas de enseñanza de docentes y los espacios de formación profesional (Unesco, 2006). En el contexto anterior, la inclusión de las TD a la educación se ha convertido en un eje dinamizador del conocimiento, mediante el cual se desarrollan los pueblos desde la perspectiva económica, política, social y ecológica. Esto supone un acercamiento continuo y permanente con las TD como medios que permiten el acceso a la información y la gestión del conocimiento (Coll, 2008).

La integración de las TD abre oportunidades y da soporte al desarrollo de un conjunto de capacidades humanas, para que las generaciones jóvenes aprendan, conozcan, se comuniquen, innoven y colaboren conforme las demandas de la sociedad actual. Sin embargo, cabe anotar que las TD no incidirán en la mejora de los aprendizajes y el desarrollo de habilidades en el estudiantado, si no se cuenta con docentes que posean una formación pedagógica, curricular y didáctica sólida que permita la planificación de situaciones de aprendizaje significativo, que las incorpore eficazmente como recursos de apoyo en el quehacer personal y profesional. Por ello, las relaciones entre las TD y los sistemas educativos constituyen un asunto crucial para el desarrollo económico, humano y la integración social de un país.

El grado de interacción con el medio que proveen las TD facilita la elaboración de nuevos significados por parte del sujeto aprendiz, mediante la estimulación e interacción simbólica de este, por medio de los recursos digitales; es así como se favorece la posibilidad de generar procesos cognitivos, que junto con los conocimientos previos permiten la compresión de los contenidos, la construcción de significados y la integración a la estructura de conocimientos que posee el individuo. Los recursos multimedia y la interactividad provista por las TD estimulan el aprendizaje significativo enmarcado dentro de la teoría cognitiva de la asimilación de Ausubel, ya que "...en el centro de la teoría de la asimilación se encuentra la idea de que los nuevos significados se adquieren mediante la interacción [característica indiscutible de las TD] de ideas (conocimientos) nuevas y potencialmente significativas con conceptos y proposiciones aprendidos con anterioridad (Ausubel, 2002, p. 171). En relación con la descripción anterior, el aprendizaje significativo 
se conceptualiza como "...una forma de aprendizaje consistente en activar experiencias y conocimientos previos con los que se relaciona e integra el nuevo conocimiento, en un proceso que implica atribución de significado o comprensión de conceptos" (Rivas, 2008, p. 28).

En el marco de una mejora del aprendizaje mediante la inserción de TD en Costa Rica, nace, como una iniciativa conjunta entre el Ministerio de Educación Pública de Costa Rica (MEP) y la Fundación Quirós Tanzi, en el año 2011, el proyecto Conectándonos, dirigido a instituciones de la educación primaria. Este utiliza las TD como una herramienta de aprendizaje, con un modelo 1:1 (una computadora por niño) (MEP y FQT, 2011). La propuesta pedagógica del proyecto Conectándonos en el ámbito educativo pretende generar indudablemente cambios en el proceso de enseñanza y aprendizaje, en los espacios de aprendizaje y, principalmente, en los papeles de los niños, niñas y personas adultas. Al considerar estas tecnologías un medio y no un fin en sí mismo, interesa cambiar la cultura de aprendizaje: de transmisión de conocimientos en el aula, a una cultura de aprendizaje inclusiva, ubicua, creativa, significativa, democrática y comunitaria (MEP y FQT, 2011).

En los últimos años el énfasis en la implementación del proyecto ha estado centrado en que cada niño o niña tenga una computadora de tipo XO y que su aprendizaje sea experiencial, autónomo, colaborativo y creativo (MEP y FQT, 2011). Asimismo, se promueve la participación en comunidades de aprendizaje, donde se facilita la comunicación, el trabajo colaborativo y el compartir asertivamente entre miembros de la comunidad educativa.

A finales del año 2015 el MEP y la Fundación Quirós Tanzi acuerdan cambiar las computadoras portátiles de tipo OLPC XO por computadoras portátiles tipo Clamshell y complementar el tipo de equipamiento que tradicionalmente se había ofrecido a las escuelas participantes en el proyecto, con proyectores multimedia interactivos. Esta decisión obedeció, en gran medida, a la implantación del Programa Nacional de Tecnologías Móviles “Tecno@ prender" en el seno de la Dirección de Recursos Tecnológicos en Educación del MEP (MEP y FQT, 2011). Todo lo anterior, a partir de la promoción de la innovación educativa y tecnológica en los procesos de enseñanza; con la propiciación de ambientes de aprendizaje significativo para facilitar, a los actores del currículo, un mayor aprovechamiento de las TD, coherente con las exigencias de la sociedad actual.

Todo lo anterior promueve que la directora de la Dirección de Recursos Tecnológicos en Educación asigne al Departamento de Investigación, Desarrollo e Implementación para generar una prueba piloto con el objetivo de explorar las prácticas pedagógicas con el uso de computadoras portátiles y un proyector interactivo, partiendo de las actividades de mediación planificadas por una persona docente destacada del proyecto y la participación de niñez de primer grado y de segundo grado, para valorar y definir su efectividad en el ambiente educativo. La situación problema que origina la prueba piloto tiene que ver con la necesidad de hacer 
doi: http://dx.doi.org/10.15359/ree.22-2.4

URL: http://www.una.ac.cr/educare

CORREO: educare@una.cr

un cambio en el equipamiento con el que inició en 2011, el proyecto Conectándonos. Por ello, se analizaron diversas opciones y, dentro de ellas, se valoró la posibilidad de introducir en los centros educativos computadoras portátiles tipo Clamshell y proyectores interactivos.

En la prueba piloto, las prácticas pedagógicas se conciben como un conjunto de estrategias de enseñanza y aprendizaje participativas promovidas por el personal docente con el uso de las TD como recursos didácticos de apoyo, con el objetivo de estimular el aprendizaje significativo y el desempeño del estudiantado mediante las actividades de mediación participativas. En este sentido cabe recalcar que "... no es suficiente el conocimiento que los docentes tienen de estas tecnologías; debe reconocerse en el contexto las potencialidades y complejidades de su relación a partir de situaciones cotidianas" (Mendoza, Buitrago y González, 2014, p. 364). De ahí la necesidad de realizar una prueba piloto donde se pueda evidenciar el nivel de interactividad que proporcionan las portátiles tipo Clamshell y el proyecto interactivo cuando se utilizan como recurso didáctico en el proceso de mediación.

Según lo antes descrito, la mediación pedagógica se determina mediante la integración de las TD en las prácticas pedagógicas desarrolladas por parte del personal docente, tanto dentro como fuera del aula, para el logro de los objetivos propuestos en los programas de estudio. Sin duda, lo anterior implicará una planificación exhaustiva de las actividades para la mediación, un conocimiento profundo sobre las posibilidades de interactividad provista por las TD y las estrategias que el personal docente promueva para un uso efectivo de las TD.

\section{Desarrollo de la propuesta}

La prueba piloto se desarrolló con un único grupo de 20 estudiantes de una escuela primaria, que pertenece a la Dirección Regional de Educación de Alajuela. El grupo de participantes estuvo compuesto por un docente líder con amplia experiencia en el proyecto, diez estudiantes de primer grado y diez estudiantes de segundo grado.

En relación con la conceptualización de maestro líder, se puede afirmar que es un actor educativo que tiene la habilidad de dinamizar el proceso de enseñanza y aprendizaje para que sus estudiantes promuevan y logren aprendizajes significativos mediante una participación activa que coadyuve no solamente al logro de los objetivos del programa de estudio, sino también al desarrollo pleno de valores y habilidades de convivencia. Por otra parte, una persona docente líder es capaz de lograr que sus aprendices se familiaricen con las nuevas ideas y recursos didácticos para el aprendizaje, a la vez que proyecta credibilidad y confianza para satisfacer sus necesidades educativas (García, 2007; Ramírez, 2004; Vázquez, Bernal y Liesa, 2014). En el mismo sentido, la escogencia del docente líder que participó en la prueba piloto se fundamentó en tres aspectos: a) poseer habilidades de convivencia que le permitieran relacionarse de manera asertiva con estudiantes y otros miembros de la comunicad educativa, b) tener sólida formación 
pedagógica y específica en las áreas disciplinares que integran el currículo, c) demostrar un alto nivel de uso, acceso y apropiación de las TD, tanto en el ámbito personal como profesional.

Como técnicas para la recolección de datos se establecieron la observación, la encuesta y la revisión documental. En relación con las observaciones fueron de tipo no participante, distribuidas a lo largo de seis días y aplicando dos observaciones cada día. En relación con lo anterior, el comité asesor del Departamento de Investigación, Desarrollo e Implementación y de la Fundación Quirós Tanzi observaron simultáneamente al docente y al grupo durante cuatro horas. El sentido de la estrategia de observación no participante consistía en tener la posibilidad de comparar, al final, los resultados y profundizar en las categorías y subcategorías propuestas. Previo al inicio de cada una de las observaciones se procedía a la revisión documental del planeamiento didáctico del docente, con el propósito de conocer los elementos de este mismo para contrastar con la información que se obtendría mediante las observaciones. Al docente líder le fue aplicado, en el último día, un cuestionario con preguntas abiertas para obtener información en relación con su experiencia durante el proceso.

Antes de iniciar la prueba piloto, se socializó la estrategia y el instrumento de observación no participante con el docente a cargo del grupo de estudiantes. Asimismo, se dio una capacitación inicial al docente sobre el uso de la computadora portátil Clamshell y el proyector interactivo. En relación con la organización del planeamiento didáctico, el docente socializó con el equipo asesor, los objetivos, los contenidos, las actividades de mediación y las estrategias de evaluación plateadas para cada una de las clases observadas durante la prueba piloto.

En cuanto al nivel de profundidad de la prueba piloto, se situó en un nivel exploratorio, que permitió el acercamiento a un conjunto de conocimientos que coadyuvan a poder acceder al desarrollo de habilidades que requieren mayor profundización conceptual (Oficina de Innovación Educativa con Uso de Nuevas Tecnologías, 2013). En este sentido, tanto docente como estudiantes, se familiarizaron con las posibilidades que ofrecía el equipo, a la vez que se provocó una reflexión sobre algunos componentes del proceso de enseñanza y aprendizaje, para responder, así, a las necesidades curriculares, a las expectativas de los sujetos participantes y a las demandas del contexto.

Por otro lado, el desarrollo de la prueba piloto implicó el planteamiento de objetivos, con el fin de formalizar las categorías y subcategorías de análisis, a saber:

\section{Objetivo general}

Explorar los beneficios que ofrecen las tecnologías digitales como recursos didácticos en el proceso de enseñanza y aprendizaje de un docente líder y sus estudiantes en una escuela de la Dirección Regional de Educación de Alajuela, durante el Plan-200 de 2015. 
doi: http://dx.doi.org/10.15359/ree.22-2.4

URL: http://www.una.ac.cr/educare

CORREO: educare@una.cr

\section{Objetivos específicos}

- Evidenciar el fortalecimiento de la mediación pedagógica mediante la utilización de las computadoras portátiles tipo Clamshell y un proyector interactivo.

- Determinar el acceso, la selección y los usos de recursos didácticos digitales contenidos en las computadoras portátiles tipo Clamshell y un proyector interactivo.

- Establecer las facilidades para la comunicación durante la mediación pedagógica provista por las computadoras portátiles tipo Clamshell y un proyector interactivo.

- Identificar las posibilidades para la búsqueda, selección, transformación y generación de información ofrecidas por las computadoras portátiles tipo Clamshell y un proyector interactivo.

Desde las consideraciones anteriores, se establecieron una serie de habilidades acordes con las tendencias dentro de los procesos de formación en los sistemas educativos mundiales, y muy particularmente, según los requerimientos y la propuesta pedagógica del Programa Nacional de Tecnologías Móviles "Tecno@prender".

Como parte de la estrategia para analizar posteriormente la información de la prueba piloto, se consideró inicialmente una metacategoría denominada "beneficios que ofrecen las TD como recursos didácticos en el proceso de enseñanza y aprendizaje" y a partir de ella, se determinaron cuatro dimensiones de la categoría fundamental relacionas con el "fortalecimiento de la mediación pedagógica", el "acceso, selección y usos de recursos didácticos digitales", las "facilidades para la comunicación" y las "posibilidades para la exploración, transformación y generación de conocimientos"(Oficina de Innovación Educativa con Uso de Nuevas Tecnologías, 2013).

Por otra parte, estas categorías fueron desglosadas en subcategorías, con el fin de establecer criterios de mayor profundidad a la hora de observar las actividades de mediación y, consecuentemente, el uso de los recursos didácticos digitales. La categorización antes descrita se presenta en la Tabla 1.

Dadas las condiciones que anteceden, en relación con la guía de observación y el cuestionario de preguntas abiertas planteados a partir de la Tabla 1, dentro de los procedimientos que se adoptaron para obtener la credibilidad de los instrumentos estuvo la estancia en el campo para su aplicación, auditoría de los asesores pedagógicos de la Fundación Quirós Tanzi, comparar con la teoría la Tabla 1 de categorías para la construcción de los instrumentos, la discusión del instrumento con los sujetos participantes, hacer un listado de las necesidades y retos del proyecto Conectándonos (Hernández, Fernández y Baptista, 2014). 
Tabla 1: Categorización propuesta para obtener información de la prueba piloto

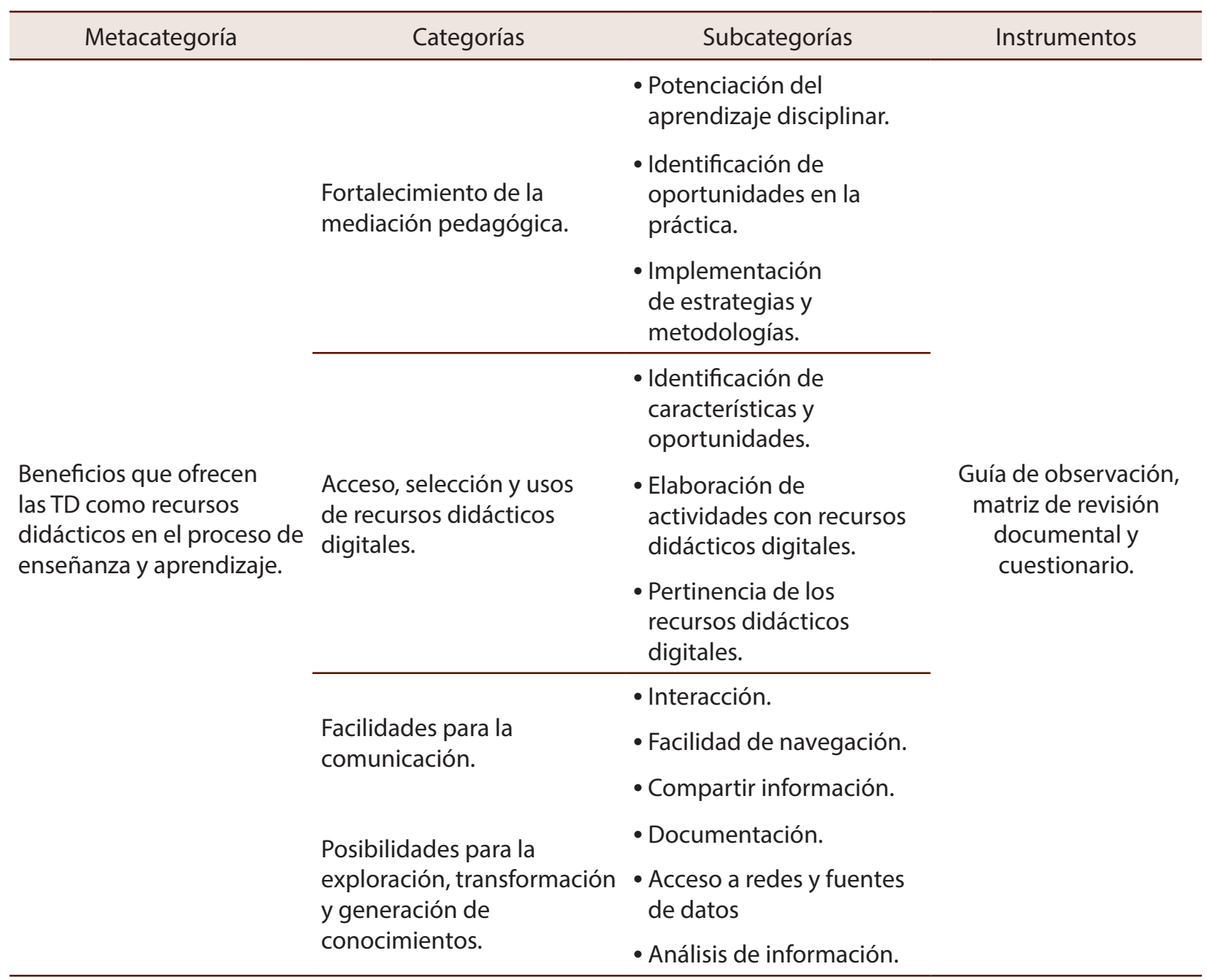

Nota: Elaboración propia.

Atendiendo los planteamientos anteriormente descritos, se sometió la guía de observación y el cuestionario al criterio de dos profesionales en asesoría pedagógica de la Fundación Quirós Tanzi y dos personas asesoras nacionales de educación del Departamento de Investigación, Desarrollo e Implementación de la Dirección de Recursos Tecnológicos en Educación del MEP. Los asesores determinaron la pertinencia de la guía de observación y del cuestionario en cuanto a la concordancia de preguntas y su relación con los alcances de la prueba piloto. Luego de la revisión, fueron consideradas las observaciones hechas por el personal asesor; entre ellas, el describir las subcategorías para facilitar el análisis y la interpretación de los datos, a saber (ver Tabla 2): 
doi: http://dx.doi.org/10.15359/ree.22-2.4

URL: http://www.una.ac.cr/educare

CORREO: educare@una.cr

Tabla 2: Descripción de las subcategorías de análisis para la prueba piloto

\section{Subcategorías}

Potenciación del aprendizaje

disciplinar

Identificación de oportunidades

en la práctica
Descripción

El equipo coadyuva efectivamente al aprendizaje y a la profundización de conocimientos y prácticas propias del área disciplinar.

Mediante la interacción con el equipo es posible la identificación de oportunidades, implicaciones y riesgos del uso del equipo.

Implementación de estrategias y El equipo coadyuva a la implementación de diversas estrategias, metodologías metodologías y seguimiento en la mediación pedagógica.

Identificación de características y oportunidades

Elaboración de actividades con recursos didácticos digitales

Pertinencia de los recursos didácticos digitales
Los recursos tecnológicos y los medios audiovisuales que posee el equipo ofrecen oportunidades para la mediación de los temas.

Los recursos tecnológicos y los medios audiovisuales permiten la creación de actividades de aprendizaje y contenidos.

El equipo posibilita la determinación de la calidad, pertinencia y la fiabilidad de los recursos didácticos digitales y medios disponibles en postales educativos, además de otros sitios en la web.

Interacción

El equipo coadyuva a la comunicación sincrónica y asincrónica entre los actores curriculares que intervienen en la mediación.

Navegación

Por medio del equipo, la navegación es eficiente y permite la utilización del internet.

Compartir información

El equipo facilita el compartir información a través de diversos canales.

Documentación de observaciones Es posible documentar las observaciones del entorno a través de los dispositivos.

Acceso a redes y fuentes de datos Mediante los dispositivos, es posible la identificación de redes, bases de datos y fuentes de información que posibilitan procesos de investigación.

Análisis de información

Los dispositivos y los recursos digitales que poseen permiten: el ordenamiento, la filtración y el análisis de la información disponible en internet.

Nota: Elaboración propia, a partir de los datos de la Oficina de Innovación Educativa con Uso de Nuevas Tecnologías (2013). 
Sobre la base de las consideraciones anteriores, una vez que fueron aplicados los instrumentos, la información fue ordenada, codificada, procesada, tabulada, representada y analizada según las metacategorías, categorías y subcategorías de la Tabla 1. Se inició con la revisión y organización del material de acuerdo con cada uno de los objetivos específicos de la prueba piloto, posteriormente se categorizaron los datos; se codificaron las categorías; se relacionaron los datos; se aplicó el procesamiento de los datos; se realizaron las representaciones gráficas de los resultados, los cuales se interpretaron y discutieron y, se obtuvieron síntesis y conclusiones en relación con las categorías y subcategorías de análisis.

\section{Análisis de los datos}

El análisis de la prueba piloto se basó en el razonamiento mediante metacategorías, categorías y subcategorías que permitieron conocer a profundidad el contexto. Para el análisis cualitativo de los datos obtenidos de las 12 guías de observación y del cuestionario fueron consideradas cuatro categorías y doce subcategorías, según la Tabla 1, posteriormente se vincularon los datos obtenidos, estableciendo criterios de coincidencia, similitudes, diferencias, presencia o ausencia de términos o frases (Salinas y Cárdenas, 2008). Por otro lado, el proceso posibilitó el establecimiento de subcategorías emergentes que no fueron consideradas inicialmente en el estudio.

\section{Fortalecimiento de la mediación pedagógica}

Para el análisis de esta categoría, se establecieron tres subcategorías. La primera de ellas relacionada con la potenciación del aprendizaje disciplinar, en la cual se requería determinar si el equipo coadyuvaba efectivamente, con el aprendizaje, y la profundización de conocimientos y prácticas propias del área disciplinar en la que se desarrolló la lección. En este sentido, las personas observadoras notaron lo siguiente: "...el equipo permitió la mediación del contenido", "...los estudiantes se encontraban trabajando en el aplicativo Paint y en Art Rage, básicamente aprendieron a usar las diferentes categorías y funciones de estas actividades " y "...las Clamshell [sic] poseen... aplicativos que pueden ser muy útiles para abordar contenidos, tales como Cmap Tools, Movie Maker, Office, entre otras". Por otra parte, el docente participante en la prueba piloto expresó: "Sí ayuda, por ser novedoso y funcional"; no obstante, señaló la importancia que las aplicaciones estén accesibles en el idioma español.

En este mismo sentido, y en relación con la segunda subcategoría, identificación de oportunidades en la práctica, considerando las oportunidades, implicaciones y riesgos del uso del equipo se observó "...que surgen dudas en los niños y niñas al utilizar el equipo y los diferentes aplicativos. Las dudas surgen en cuanto al uso de las aplicaciones". Por otro lado, se escuchó decir a los niños ".. las compus se parecen al celular". Asimismo, el docente a cargo del grupo manifestó que el equipo "...facilita enormemente la interacción de los estudiantes..." a la vez que coadyuva al desarrollo de un "mayor interés, hay expectativa, deseo por aprender". 
doi: http://dx.doi.org/10.15359/ree.22-2.4

URL: http://www.una.ac.cr/educare

CORREO: educare@una.cr

En el orden de las ideas anteriores, en relación con la tercera subcategoría, implementación de estrategias metodológicas, en donde se observó si el equipo coadyuvaba a la implementación de diversas estrategias, metodologías y seguimiento en la mediación pedagógica, se determinó en la observación que el docente "aplicó estrategias en las cuales exploraban usando internet, creaban usando aplicativos como Movie Maker y Paint y programaron con la actividad Tortugarte". Asimismo, se observó que "el equipo contiene diferentes categorías de actividades que permiten explorar, construir y programar según el tema que se encuentren trabajando". Así, fue posible establecer mediante la observación que efectivamente el equipo coadyuvó al ofrecimiento de oportunidades para la mediación de los temas, ya que según se anotó "...utilizaron [estudiantes] la cámara y un programa de edición de video para crear sus propias producciones". Por otra parte, el docente manifestó que "...por ser un equipo nuevo se dificulta en partela manipulación yen ocasiones el acceso a algunas aplicaciones", por ello, propone la ampliación del proceso de inducción para la medicación pedagógica con los dispositivos que ofrece el equipo asesor. Para finalizar, se presenta la información relevante relacionada con esta variable por medio de la Figura 1.

\begin{tabular}{|c|c|c|}
\hline \multicolumn{3}{|c|}{ Potenciación del aprendizaje disciplinar } \\
\hline \multirow{6}{*}{$\begin{array}{l}\text { - El equipo permitió la mediación } \\
\text { del contenido. } \\
\text { - Las Clamshell poseen ... } \\
\text { aplicativos que pueden ser muy } \\
\text { útiles para abordar contenidos. } \\
\text { - Si ayuda por ser novedoso y } \\
\text { funcional. } \\
\text { - Es necesario que las aplicaciones } \\
\text { estén en idioma español. }\end{array}$} & \multicolumn{2}{|c|}{ Identificación de oportunidades en la práctica } \\
\hline & \multirow{6}{*}{\begin{tabular}{|l} 
- Surgen dudas en el \\
estudiantado al interarctuar \\
con el equipo y los diferentes \\
aplicativos. \\
- Facilita enormemente la \\
interacción estudiantil. \\
- Coadyuva al desarrollo de un \\
"mayor interés, hay expectativa, \\
deseo por aprender.
\end{tabular}} & $\begin{array}{l}\text { Implementación de estrategias y } \\
\text { metodologías }\end{array}$ \\
\hline & & \multirow{6}{*}{$\begin{array}{l}\text { - El maestro aplicó estrategias en } \\
\text { las cuales exploraban, usando } \\
\text { internet, creaban usando } \\
\text { aplicaciones. } \\
\text { - Se dificultó en parte la } \\
\text { manipulación, y en ocasiones, el } \\
\text { acceso a algunas aplicaciones. } \\
\text { - Es necesario ampliar el proceso } \\
\text { de inducción. }\end{array}$} \\
\hline & & \\
\hline & & \\
\hline & & \\
\hline & & \\
\hline & & \\
\hline
\end{tabular}

Figura 1: Análisis de la categoría "fortalecimiento de la mediación pedagógica".

Nota: Elaboración propia.

\section{Acceso, selección y usos de recursos digitales}

Referente a la clasificación de la Tabla 1, para el análisis de esta categoría se consideraron de igual manera tres subcategorías. La primera de ellas relacionada con la identificación de características y oportunidades que ofrecía el equipo. En este sentido, se quiso conocer si los recursos tecnológicos y los medios audiovisuales de los equipos digitales usados en la prueba piloto ofrecían oportunidades para la mediación de los temas desarrollados en la clase. Los observadores indicaron que "... los diferentes aplicativos ofrecen opciones variadas para abarcar distintos temas o asignaturas. Asimismo, "... se observa que muchos aplicativos son funcionales para construir o crear". Por último, el docente manifestó que "los equipos son excelentes, modernos y prácticos, muy funcionales para los estudiantes", y ofrecen la "... oportunidad y posibilidad para la mediación de los temas propuestos".

Manuel Baltodano-Enríquez

Los artículos de la Revista Electrónica Educare del Centro de Investigación y Docencia en Educación de la Universidad Nacional, Costa Rica, se comparten bajo términos de la Licencia Creative Commons: Reconocimiento, № Comercial, Sin Obra Derivada 3.0 Costa Rica. Las autorizaciones adicionales a las aquí delimitadas se pueden obtener en el correo: educare@una.cr 
En relación con la subcategoría, elaboración de actividades con recursos digitales, donde se observó si los recursos tecnológicos y los medios audiovisuales permitín la creación de actividades de aprendizaje y contenidos, se pudo constatar que, efectivamente, los recursos tecnológicos y los medios audiovisuales permitieron la creación de actividades de aprendizaje y contenidos. Al respecto, se rescató "... el aplicativo Paint, elaborando y editando dibujos sobre el tema Vacaciones". Por su parte, el docente manifestó que dichos recursos digitales favorecieron "...la creación de actividades de aprendizaje, floreciendo la práctica, la creatividad y la interacción con el equipo".

Finalmente, en relación con la tercera subcategoría: pertinencia de los recursos digitales, se quiso evidenciar si el equipo posibilita la determinación de la calidad, pertinencia y la fiabilidad de los recursos didácticos y medios disponibles en portales educativos y otros sitios de la web. Al respecto, fue evidente que el equipo posibilitó la "...determinación de la calidad, pertinencia y la fiabilidad de los recursos y medios disponibles en postales [sic] educativos y otros sitios de la web". Asimismo, el docente instruyó a sus estudiantes para que realizaran búsquedas de imágenes y otros recursos. En relación con lo anterior, el docente manifestó que "...la calidad de las imágenes, la accesibilidad, la trasferencia de imágenes y videos web es efectiva"; en otras palabras, los recursos didácticos digitales obtenidos en la web se observan y escuchan con alta calidad en las computadoras portátiles Clamshell y el proyector interactivo. Asimismo, el equipo permitió a estudiantes "guardary trasferir imágenes". Para finalizar, los resultados se resumen en la Figura 2.

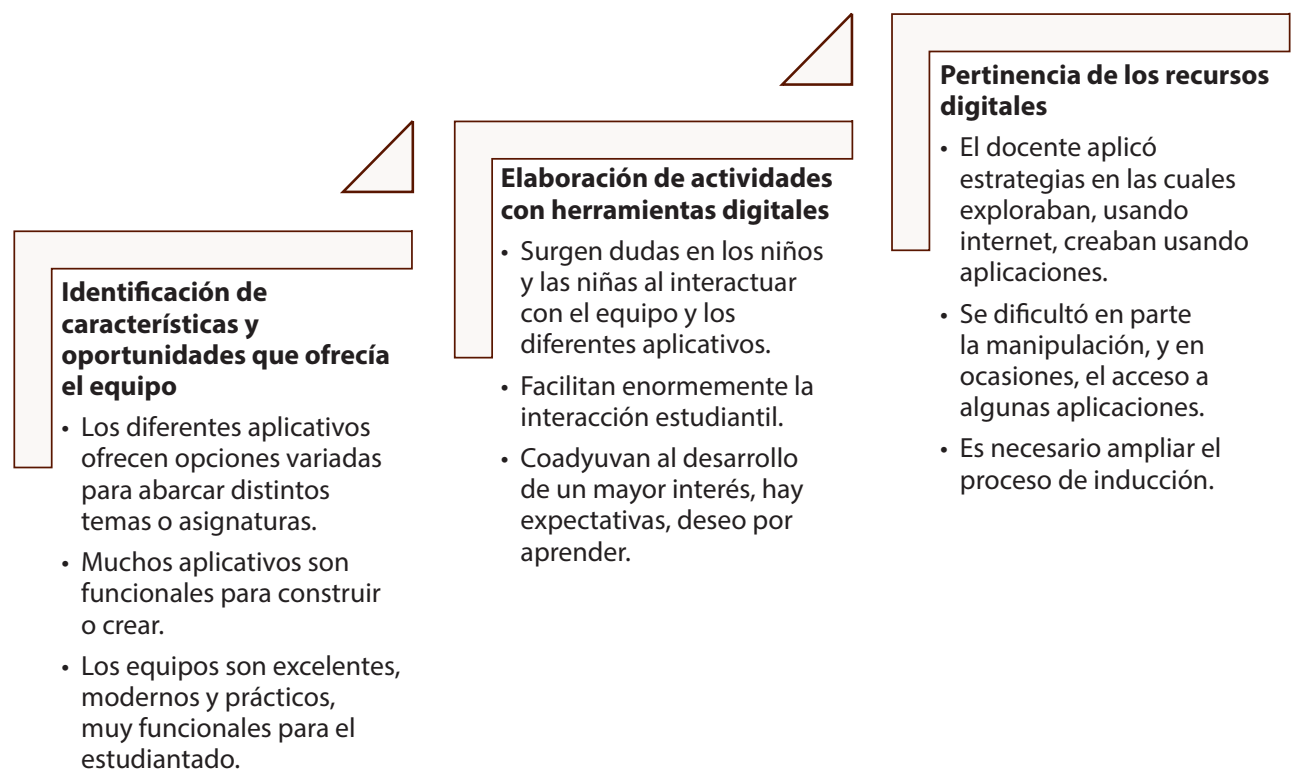

Figura 2: Análisis de la categoría "acceso, selección y usos de recursos digitales".

Nota: Elaboración propia. 
doi: http://dx.doi.org/10.15359/ree.22-2.4

URL: http://www.una.ac.cr/educare

CORREO: educare@una.cr

\section{Facilidades para la comunicación}

En relación con la tercera categoría, se consideraron tres subcategorías; la primera de ellas relacionada con la interacción, en la cual se observó si el equipo coadyuvaba a la comunicación sincrónica y asincrónica entre los actores curriculares que intervienen en la mediación. Según lo anterior, en la guía de observación se anotó que“...losylas estudiantes tiene [sic] interacción entreellos, se explican entre ellos lo que no comprenden y hay comunicación constante entre docente - estudiante también". Asimismo, se observó que "los niños pudieron compartir sus trabajos con la capacidad que tiene el proyector ... para proyectar las pantallas de varias computadoras". Por otra parte, el docente se mostró complacido de tener la opción de poder monitorear el trabajo de sus estudiantes desde su ordenador, por ello, opinó que el equipo es muy efectivo en relación con este aspecto; sin embargo, resalta la importancia de tener capacitación y prácticas más prolongadas con el equipo.

En cuanto a la segunda subcategoría: navegación, se observó si por medio del equipo la navegación era eficiente y permitía la utilización del internet de manera fluida; al respecto el equipo observador registró que no hubo inconveniente para acceder de manera fluida al internet. Según la opinión del docente, la navegación posibilitó a sus estudiantes el "...acceso rápido, ágil y oportuno" a la información.

Por último, en cuanto a la tercera subcategoría se observaron las posibilidades ofrecidas por el equipo para compartir información a través de diversos canales. En relación con lo anterior, fue posible observar cuando los niños y las niñas compartieron entre sí y con el docente sus producciones por medio del proyector interactivo y dispositivos USB. De la misma forma, el docente afirmó que, durante el proceso de inducción, se pudieron conocer diversos programas que permiten el compartir información, tanto de manera sincrónica como asincrónica. Los resultados relacionados con las subcategorías anteriores se resumen en la Figura 3.

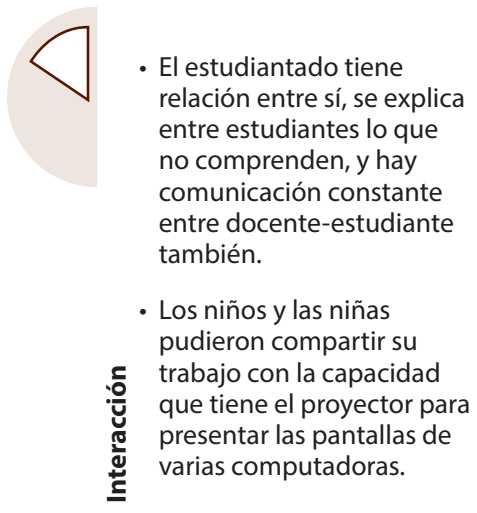

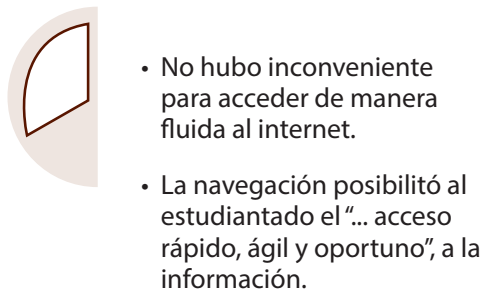

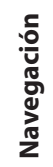

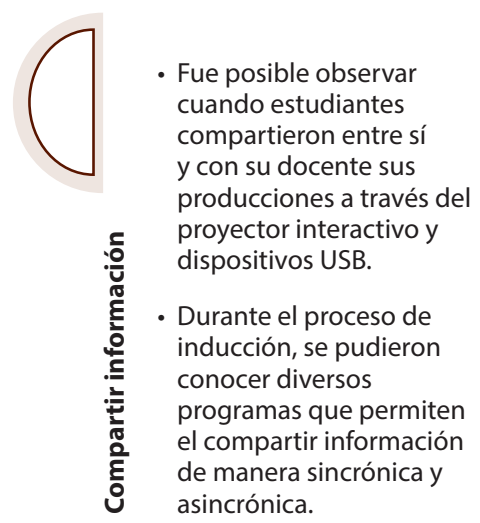
y con su docente sus producciones a través del dispositivos USB.

Durante el proceso de inducción, se pudieron conocer diversos programas que permiten de manera sincrónica y asincrónica.

Figura 3: Análisis de la categoría "facilidades para la comunicación". Resultados de las guías de observación y el cuestionario aplicado durante la prueba piloto.

Nota: Elaboración propia. 


\section{Posibilidades para la exploración, transformación y generación de conocimientos}

Para finalizar, se analizó la información obtenida en relación con la tercera y la cuarta categoría, al igual que en las categorías anteriores. Para su análisis se establecieron tres subcategorías. La primera subcategoría tenía que ver con la posibilidad de poder documentar información por medio de los dispositivos. En relación con lo anterior, el docente manifestó que "...tanto los estudiantes como el docente logran documentar todo, guardando la información tanto en la computadora como en dispositivos externos". Asimismo, se observó que "los niños tomaron fotografías de su contexto para elaborar un proyecto con un tema propuesto por el profesor". El hecho de que el tema fuera propuesto por el profesor obedeció a una intención evaluativa particular, ya que la temática había sido abordada por el docente previamente con otro grupo de estudiantes de primer grado, utilizando los recursos digitales contenidos en las computadoras de tipo OLPC XO, por eso, el maestro quiso comparar el nivel de interacción provisto por los recursos digitales de las computadoras tipo Clamshell, con el nivel de interacción obtenido en la experiencia previa con las computadoras tipo OLPC $\mathrm{XO}$, para evidenciar, de esta forma, las posibilidades y beneficios que el equipo utilizado en la prueba piloto ofrecía. Todo lo anterior, sin prejuicio de la independencia cognitiva que sus estudiantes pudieron alcanzar mediante las múltiples actividades de mediación que el maestro líder planificó previamente.

En relación con la segunda subcategoría: acceso a redes y fuentes de datos, fue evidente cuando los niños y las niñas se conectaron rápidamente ".. a Internet para la búsqueda de imágenes". Asimismo, ".. algunos observaron videos y utilizaron juegos en línea". Lo anterior es ratificado cuando los niños y las niñas "... lograron identificar las redes de internet y acceder a las mismas, además en los tiempos libres algunos exploraban páginas como Youtube [sic]". En este mismo sentido, el docente encuestado ratificó la manera ágil y rápida en la que lograron identificar redes y fuentes de datos adecuadas a las necesidades personales y requerimientos de la actividad.

Por último, en relación con la subcategoría, análisis de la información, donde se observó cómo los dispositivos y los recursos digitales permitían el ordenamiento, la filtración y el análisis de la información disponible en internet, se pudo constar que la niñez pudo descargar y seleccionar la información, además, realizar el proyecto que el docente asignó. En concordancia con lo antes descrito, el docente afirmó que "... los estudiantes en forma práctica y ágil disponen de información oportuna en la red y poseen la capacidad de guardar y filtrar la documentación requerida". Los resultados relacionados con las subcategorías anteriores se resumen en la Figura 4. 
doi: http://dx.doi.org/10.15359/ree.22-2.4

URL: http://www.una.ac.cr/educare

CORREO: educare@una.cr

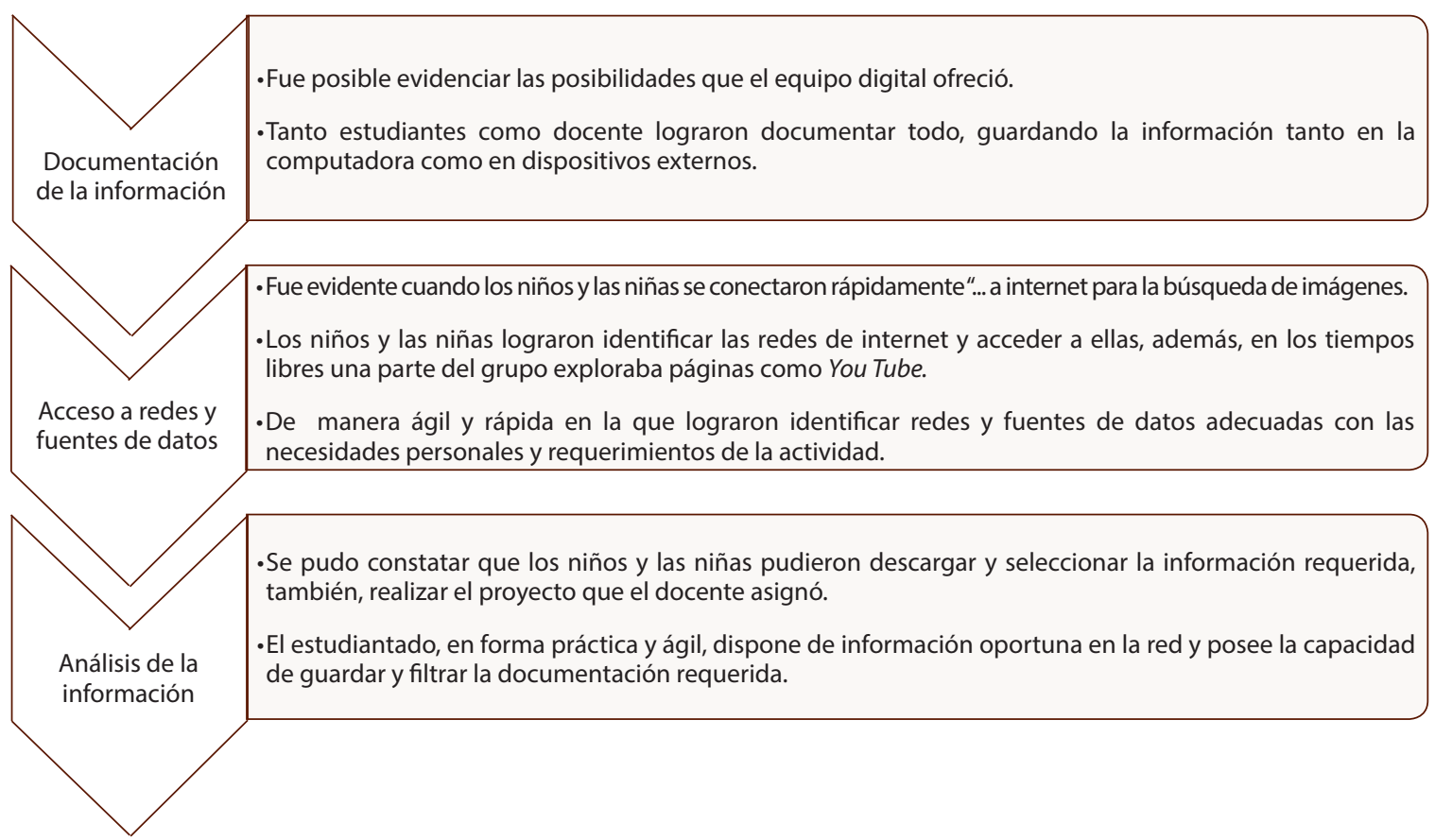

Figura 4: Análisis de la categoría "posibilidades para la exploración, transformación y generación de conocimientos". Resultados de las guías de observación y el cuestionario aplicado durante la prueba piloto.

Nota: Elaboración propia del autor.

Para dar continuidad al análisis anterior, es necesario considerar algunos elementos señalados por el maestro que participó en la prueba piloto, el cual comentó sobre las características del equipo, resaltando que es "... liviano, ergonómico, llamativo para el estudiante". Asimismo, comentó que pudo observar cómo "...los discentes valoran el equipo con mayor interés y responsabilidad, quizás por ofrecer una estructura física diferente a la que están acostumbrados a utilizar". En este sentido, hizo especial énfasis en la característica "touch" de los equipos digitales que los hace de "...un gran atractivo, algo nuevo y llamativo", para los niños y las niñas.

\section{Interpretación}

La educación se ha convertido en eje dinamizador del conocimiento mediante el cual se desarrollan los pueblos desde la perspectiva económica, política, social y ecológica. Esto supone un acercamiento continuo y permanente de los sistemas educativos con las TD, como medios que permiten gestionar el recurso más valioso del siglo XXI: el conocimiento (Coll, 2008). 
Todo lo anterior ha provocado un particular interés en relación con los procesos para el mejoramiento de la enseñanza y el aprendizaje. Desde esta perspectiva, se pone en evidencia la integración, el uso y la apropiación de las TD en los sistemas escolares, cuyas experiencias y resultados han sido variados y complejos debido a las diferencias culturales, económicas y educativas. Es por ello que los pilotos han evolucionado de la cuantificación de computadores o de estudiantes y docentes con acceso a la tecnología hacia la necesidad de conocer los eventos que subyacen en el mejoramiento cualitativo de la enseñanza y de los aprendizajes mediados con TD (De Pablos, Cólas, y González, 2010).

La descripción anterior platea que no es necesariamente en las TD donde radica el proceso de mejora continua en la enseñanza y aprendizaje, sino más bien en las actividades que lleva a cabo el personal docente para el fortalecimiento de la mediación pedagógica a partir de la potenciación del aprendizaje disciplinar la identificación de oportunidades en la práctica y la implementación de estrategias y metodologías. En este aspecto, el profesorado y estudiantado que participó en la prueba piloto pudieron experimentar con el equipo que ciertamente favoreció la medicación pedagógica.

Por otra parte, el acceso, la selección y los usos de recursos digitales favorecieron la identificación de características y oportunidades de mejora del proceso de enseñanza, mediante la elaboración de actividades con recursos digitales contenidos en las computadoras portátiles tipo Clamshell y el proyector interactivo. En relación con lo anterior, aunque ha existido una mejora en la dotación de equipos e internet en los centros escolares, aún hace falta mayor cobertura y un cambio de las prácticas de los maestros y las maestras.

Todo lo anterior plantea la necesidad de conocer las características concretas del contexto y los perfiles de docentes con quienes se desea implantar el proceso. Desde la perspectiva anterior, la caracterización de perfil socioeducativo de estudiantes es fundamental para garantizar los medios de aprendizaje y las estrategias didácticas (Salinas, 2008).

Actualmente, existe un consenso generalizado en relación con el nuevo énfasis que requieren el uso de las TD, este consenso plantea la necesidad de generar los mecanismos que faciliten el desarrollo de habilidades para el aprendizaje y la adaptación de los individuos y las organizaciones a los nuevos tiempos (Coll, 2008; Correa y De Pablos, 2009; De Pablos et al., 2010; Fullan, 2002; Fullan y Stiegelbauer, 1997; Salinas, 2008). Así las cosas, es inminente que aquellos proyectos educativos que no trasciendan estos límites se verán obligados a realizar cambios o a quedar relegados a prácticas que en este siglo no encajan con el orden educativo.

Lo anterior supone la generación de innovaciones educativas tendientes al fortalecimiento de la enseñanza, a través de medios didácticos que faciliten el proceso de interacción colectiva y la construcción de conocimiento, que permitan cambios estructurales en la cultura del aula y de la escuela. Todo esto plantea, también, la necesidad de generar acciones para atender el 
doi: http://dx.doi.org/10.15359/ree.22-2.4

URL: http://www.una.ac.cr/educare

CORREO: educare@una.cr

desajuste que existe entre el nivel de uso y apropiación que muestran docentes y estudiantes de las TD; así como también, el desfase entre la actitud positiva y la valoración hacia las tecnologías que muestra una parte de las maestras y los maestros, y el escaso uso que hacen de estas, en su práctica educativa (Coll, 2008).

Para la mejora de los aspectos descritos anteriormente, es necesario, en primer lugar, considerar el equipamiento de los centros educativos; en segundo lugar, es fundamental el diseño pedagógico o instruccional de las actividades que incluye: contenidos, objetivos, estrategias de aprendizaje; $y$, en tercer lugar, hay que establecer los mecanismos que conducen a las interacciones de quienes participan con el modelo de diseño y las actividades propuestas y de cómo, a través de las TD, se contribuye al desarrollo de estas (Coll, 2008).

Todo lo anterior supone una mejora en la incorporación de las TD como mediadoras entre estudiantes y las tareas del aprendizaje; como instrumentos de representación y comunicación que den sentido y significado a las tareas; como instrumentos de seguimiento y evaluación de las actividades de mediación y como instrumentos de construcción de espacios interactivos para la socialización y el trabajo conjunto (Coll, 2008; Salinas, 2008).

En cuanto a las facilidades para la comunicación que ofrecieron los equipos digitales, fueron muy claras las posibilidades de interacción, la facilidad para la navegación y el compartir información que orientó el proceso de enseñanza y aprendizaje. Esto, sin duda, favoreció el aprendizaje, con actividades diversas e interacción del estudiantado en diferentes dimensiones: estudiante-objeto de conocimiento, estudiante-estudiante, estudiante-centro educativo, estudiante-consigo mismo o consigo misma (Centro de Capacitación y Educación a Distancia, 2013; Mortera, 2002).

Las características anteriormente citadas resultan fundamentales en la comprensión del papel que le corresponde jugar al personal docente. No solamente se debe ser un personal educador pedagogo y con vocación por la docencia, sino también, ser consciente de la importancia de la enseñanza y el aprendizaje a través de medios de comunicación didáctica, sean estos sincrónicos o asincrónicos. De aquí, surge la necesidad de implementar una mediación pedagógica flexible que promueva la diversificación de medios de comunicación para el logro de actividades de aprendizaje colaborativas.

Para finalizar, en cuanto a las posibilidades para la exploración, transformación y generación de conocimientos mediante la documentación, el acceso a redes, a las fuentes de datos y el análisis de información posibilitó la concreción de actividades indagatorias apoyadas con el uso de las TD, las cuales se nutren de innumerables posibilidades para el aprendizaje. Desde este paradigma, se redefinen las actividades de mediación para centrar su foco en el estudiantado como ser integral, que requiere ser estimulado para generar conocimiento y superar los problemas cotidianos. 
Al diseñar y desarrollar estrategias de enseñanza y aprendizaje que permitan el ordenamiento, la filtración y el análisis de información con el uso de TD se favorece la generación de conocimiento, asimismo, el ofrecimiento de una visión integral y novedosa sobre el uso y acceso a las TD que puede darse entre el estudiantado y el docente líder. Lo anterior es significativo, puesto que plantea la realización de un proceso de reflexión sobre las formas para apoyar el desarrollo de la clase y el ofrecimiento de una educación de calidad, que incentive la búsqueda, el tratamiento y la trasformación de la información disponible.

\section{Conclusiones y reflexiones finales}

El desarrollo e implementación de propuestas que promuevan cambios de equipo en proyectos pedagógicos requiere de estrategias que permitan evidenciar el logro de los objetivos de aprendizaje. Por ello, es preciso considerar aspectos relacionados con la conectividad, el acceso a los recursos didácticos digitales, el ambiente que facilite la interacción y el desarrollo de la mediación pedagógica, al igual que un diseño metodológico que permita la recolección de información válida y confiable.

El proceso previo de validación con personal profesional involucrado en el proyecto es determinante para garantizar la fiabilidad de los datos obtenidos y la posibilidad de observar en el contexto escolar las actividades de mediación desarrolladas por los maestros y las maestras. En efecto, el planeamiento didáctico constituye la base fundamental para la consecución del proceso. Asimismo, los cuerpos docentes requieren una preparación previa y familiarización con los dispositivos para poder gestionar las actividades de mediación que permitan a sus estudiantes el logro de los objetivos planteados y una evaluación que facilite evidenciar las mejoras alcanzadas. Por esta razón, cuando se incorporan TD, la evaluación de los aprendizajes debe concebirse como "un proceso continuo de recopilación de información cualitativa y cuantitativa, que fundamenta la emisión de juicios de valor y la toma de decisiones para la mejora progresiva del proceso de enseñanza y aprendizaje" (Departamento de Evaluación de los Aprendizajes, 2016, diapositiva 10).

De acuerdo con lo anterior, la evaluación de los aprendizajes durante la prueba piloto debe ser integral y continua para determinar el logro de los aprendizajes por parte del estudiantado; debe valorar las condiciones iniciales, intermedias y finales del proceso desde el enfoque cualitativo y cuantitativo. Cabe agregar, en este sentido, que los recursos didácticos digitales se concierten en medios de apoyo en el quehacer de los maestros y las maestras para lograr aprendizajes significativos en la niñez, para lo cual se deben conocer previamente las posibilidades de los materiales, las características socioculturales del grupo de discentes, y las funciones que desempeñan según las actividades de mediación y de evaluación planificadas por el personal docente. 
doi: http://dx.doi.org/10.15359/ree.22-2.4

URL: http://www.una.ac.cr/educare

CORREO: educare@una.cr

El cambio de los equipos digitales es un elemento que debe ser considerado en todo proyecto educativo que inserte TD como apoyo al currículo, atendiendo las tendencias mundiales y los cambios acelerados que sufren las TD. De ahí, la importancia que tiene el que las instancias involucradas promuevan acciones que permitan a los actores involucrados detectar e informar cuando el equipo utilizado ha entrado en obsolescencia o no satisface las necesidades educativas.

Lo anteriormente descrito tiene como propósito la mejora de la realidad existente, mediante la motivación y la participación de todos los actores involucrados. Las TD cambian vertiginosamente, lo que provoca que los requerimientos y demandas de la población meta sean cada vez mayores. Cuando se hace mención a la mejora de la realidad existente, es importante considerar dos aspectos: el primero está relacionado con quienes se beneficiarán del cambio y el segundo se relaciona con la solidez técnica del cambio. A partir de lo anterior, Drucker (1997) afirma que estas fuentes de cambio generan regularmente dos problemas"...la conveniencia de las innovaciones que se introducen, y la inclinación a la negligencia con respecto a los cambios necesarios" (p. 27). Por ello, es importante concientizar a los grupos docentes y a estudiantes acerca de que el cambio en el equipo tecnológico representa un insumo para la generación de mejores experiencias de aprendizaje y el acercamiento a actividades de mediación más ricas en cuento a la interacción con los objetos de aprendizaje.

Por último, la permanencia prolongada del equipo organizador de la prueba piloto es fundamental; esto permite tener criterios sustantivamente vinculados con las necesidades e intereses de las personas. De esta forma, se apoya al personal docente no solamente en el proceso de inducción para el uso, el acceso y la apropiación del equipo, sino también en la generación de estrategias, métodos y técnicas para la enseñanza y el aprendizaje, que permitan hacer reflexión sobre: ¿cómo enseñar y cómo aprender?

\section{Referencias}

Ausubel, D. P. (2002). Adquisición y retención del conocimiento. Una perspectiva cognitiva. Barcelona: Paidós. Recuperado de https://issuu.com/luisorbegoso/docs/ausubel adquisicion $y$ retencion $\mathrm{d}$

Centro de Capacitación y Educación a Distancia. (2013). Unidad 3: El diseño curricular en la UNED (Curso de formación docente: Pedagogía Universitaria). San José, Costa Rica: Euned. Manuscrito inédito.

Coll, C. (2008). TIC y prácticas educativas: Realidades y expectativas. En Fundación Santillana (Eds.), Las tecnologías de la información y la comunicación (TIC) en la educación: Retos y posibilidades (pp. 163-181). Madrid: Santillana.

Correa,J.M.y DePablos, J.(2009). Nuevas tecnologías einnovación educativa. RevistadePsicodidáctica, 14(1), 133-145. Recuperado de http://www.redalyc.org/articulo.oa?id=17512723009 
De Pablos, J., Cólas, P. y González, T. (2010). Factores facilitadores de la innovación con TIC en los centros escolares. Un análisis comparativo entre diferentes políticas educativas autonómicas. Revista Educación, 352, 23-51. Recuperado de http://www.revistaeducacion. educacion.es/re352/re352 02.pdf

Departamento de Evaluación de los Aprendizajes. (2016). Evaluación de los aprendizajes. Presentado en reunión de la Comisión Interinstitucional de Reforma Integral al Reglamento de Evaluación de los Aprendizajes [Documento inédito en archivo digital PowerPoint]. Misterio de Educación Pública, San José, Costa Rica.

Drucker, P. (1985). Fuentes de cambio educativo. En. M. G. Fullan y S. Stiegelbauer (Eds.), El cambio educativo: Guía de planeación para maestros (pp. 25-35). México: Trillas.

Fallas, I.V. y Trejos I. (2013). Educación en la sociedad de la información y el conocimiento. San José, Costa Rica: Euned.

Fonseca, C. (2000). Aprendizaje y tecnologías digitales ¿novedad o innovación? Red digital: Revista de Tecnologías de la Información y Comunicación Educativas, 1.

Fullan, M. (2002). El significado del cambio educativo: Un cuarto de siglo de aprendizaje. Profesorado, revista de currículum y formación del profesorado, 6(1-2), 1-14. España: Universidad de Granada. Recuperado de http://www.ugr.es/ recfpro/rev61ART1.pdf

Fullan, M. y Stiegelbauer, S. (1997). El cambio educativo: Guía de planeación para maestros. México: Trillas.

García, M. (2007). Nosotros, los profesores: Breve ensayo sobre la tarea docente. Madrid: UNED.

Hernández, R., Fernández, C. y Baptista, P. (2014). Metodología de la investigación (6ªed.). México: McGraw-Hill.

Mendoza, E., Buitrago, W. y González, L. F. (2014). Construcciones críticas sobre las prácticas pedagógicas: Reflexiones a partir dela psicología cultural y discursiva.Estudios Pedagógicos, 40(1), 359-372. doi: https://doi.org/10.4067/S0718-07052014000100021

Ministerio de Educación Pública. (2010). Propuesta curricular y pedagógica para la incorporación de las TIC en tercer ciclo y educación diversificada (Consultoría Dirección de Recursos Tecnológicos en Educación). San José, Costa Rica. Manuscrito inédito.

Ministerio de Educación Pública y Fundación Quirós Tanzi (FQT). (2011). Proyecto Conectándonos. Versión Adobe PDF. San José, Costa Rica: Departamento de Investigación, Desarrollo e Investigación. Dirección de Recursos Tecnológicos en Educación. Manuscrito inédito.

Mortera, F. (2002). Educación @ distancia y diseño instruccional: Conceptos básicos, historia y relación mutua. México: Taller Abierto. 
doi: http://dx.doi.org/10.15359/ree.22-2.4

URL: http://www.una.ac.cr/educare

CORREO: educare@una.cr

Oficina de Innovación Educativa con Uso de Nuevas Tecnologías. (2013). Competencias TIC para el desarrollo docente. Colombia: Ministerio de Educación Nacional. Recuperado de http:// www.colombiaaprende.edu.co/html/micrositios/1752/articles-318264 recurso tic.pdf

Organización de las Naciones Unidas para la Educación la Ciencia y la Cultura. (2004). Las tecnologías de la información y la comunicación en la formación docente: Guía de planificación. Montevideo, Uruguay: Ediciones Trilce. Recuperado de http://unesdoc. unesco.org/images/0012/001295/129533s.pdf

Organización delas Naciones Unidas parala Educación, la Cienciayla Cultura.(2006). Laintegración de las tecnologías de la información y la comunicación en los sistemas educativos: Estado del arte y orientaciones estratégicas para la definición de políticas educativas en el sector. París: Autor. Recuperado de http://unesdoc.unesco.org/images/0015/001507/150785s.pdf

Ramírez, N. (2004). Experiencia de una docente líder que impulsa el cambio estructurando una visión compartida y coligando potencialidades. Educere, 8(24), 77-82. Recuperado de http://www.redalyc.org/articulo.oa?id=35602413

Resnick, M. (2000). It's not just information. IBM Systems Journal, 39(3.4), 816-817. doi: https:// doi.org/10.1147/sj.393.0816

Rivas, M. (2008). Procesos cognitivos y aprendizaje significativo. Madrid: Comunidad de Marid, Consejería de Educción. Recuperado de http://www.deposoft.com.ar/repo/publicaciones/ A9R6652.pdf

Salinas, J. (2008). Innovación educativa y usos de las TIC. Sevilla: Universidad Internacional de Andalucía. Recuperado de http://gte.uib.es/pape/gte/sites/gte.uib.es.pape.gte/files/ innovac tic salinas1.pdf

Salinas, P. y Cárdenas, M. (Eds.). (2008). Métodos de investigación social: Una aproximación desde las estrategias cuantitativas y cualitativas ( $2^{2}$ ed.). Antofagasta, Chile: Universidad Católica del Norte.

Vázquez, S., Bernal, J. L. y Liesa, M. (2014). La conceptualización del liderazgo: Una aproximación desde la práctica educativa. REICE. Revista Iberoamericana sobre Calidad, Eficacia y Cambio en Educación, 12(5), 79-97. Recuperado de http://www.redalyc.org/articulo. oa?id=55132460004 